\title{
Epidemiological Characteristics of Infections Associated with Culture Isolated Fusobacterium Nucleatum: A Retrospective Analysis
}

\author{
Shah C, Moolani Y and Campbell S \\ Baylor College of Medicine, Texas, USA
}

*Corresponding author: Campbell S, Baylor College of Medicine, Texas, USA, Tel: (317) 504-5759, Office:

(713) 798-6724, E-mail: steffanie.campbell@bcm.edu

Citation: Shah C, Moolani Y, Campbell S (2015) Epidemiological Characteristics of Infections Associated with Culture Isolated Fusobacterium Nucleatum: A Retrospective Analysis. J Case Rep Stud 3(1): 103. doi: $10.15744 / 2348-9820.2 .403$

Received Date: October 03, 2014 Accepted Date: January 30, 2015 Published Date: February 03, 2015

\begin{abstract}
Objectives: The pathogenic potential of Fusobacterium nucleatum and its significance in infections has gained new interest. This retrospective study assessed the epidemiology of patients with infections and culture isolated F. nucleatum at two county hospitals in Houston, Texas.

Methods: Clinical, epidemiological and microbiological data were obtained from the electronic medical record for both Ben Taub General Hospital and Lyndon B. Johnson Hospital from January 1, 2002 to December 31, 2011.

Results: The overall incidence of patients with culture isolation of F. nucleatum associated was $0.0113 \%$ (45 patients out of approximately 400,000 patient admissions). Skin and soft tissue infections were the most prevalent (31\%). In our study, only $6.3 \%$ were immunocompromised. Males represented $66.7 \%$ of the subjects. Our median age was much lower than previous reports at 43.4 years. $F$. nucleatum was isolated from a wound or abscess in $77.5 \%$ and $100 \%$ of those patients required surgical intervention. Of the 40 patients identified, 38 (95\%) survived at least 30 days after discharge from the hospital.

Conclusions: In our report of 40 cases, skin and soft tissues were the most frequent sites of $F$. nucleatum isolation. Most infections were related to a wound or abscess that needed surgical intervention in addition to antibiotics. No significant association was noted with an immunocompromised state.
\end{abstract}

\section{Introduction}

Fusobacterium species are obligate anaerobic, Gram-negative bacilli which inhabit the oral, gastrointestinal, upper respiratory and genito-urinary tract as part of the normal flora [1]. Although F. nucleatum comprise a significant component of the human normal flora they can also cause a variety of usually endogenous infections [2-7]. F. nucleatum infections have been associated with increased morbidity and mortality [8-11]. F. nucleatum are usually isolated from a wide variety of clinical specimens [9-12]. Because of the association with upper respiratory tract flora, they are frequently isolated from chest infections, including aspiration pneumonia, lung abscess and empyema [5,13].

The pathogenic potential of F. nucleatum and its significance in infections has gained new interest for several reasons. First, this bacterium has the potential to be pathogenic because of its frequency in periodontal lesions and the now recognized impact of oral disease on overall health [14-16]. It also has been identified as a producer of tissue irritants and is known to have synergism with other bacteria in mixed infections [17]. Lastly, cloning and genetic sequencing have allowed a better understanding of the structure and function of the outer membrane proteins produced by Gram-negative bacteria, including F. nucleatum which is involved in this noted synergy [1].

F. nucleatum is a notoriously difficult species to cultivate; consequently, microbiological investigation and susceptibility testing is considered laborious and often times F. nucleatum is identified long after the patient is discharged from the hospital [18,19]. Because of these difficulties, PCR-based methods and other molecular methods such as DNA-DNA hybridization have been developed but have not been implemented at our facility as of yet [20]. Thus, antimicrobial therapy is usually empiric. Recently fusobacteria has been demonstrated to have reduced susceptibility or resistance to some of the commonly used empiric antibiotics, such as trimethoprim/sulfamethoxazole (TMP-SMX) and clindamycin, making early identification more important [21-23].

The majority of previously-published studies have been conducted in tertiary care centers and with selected patient populations, including immunocompromised and cancer patients [24]. The risk factors and characteristics of F. nucleatum infections have rarely been described in a non-selected population. Estimates of the incidence of these infections have been complicated by their rarity, difficulty in culture isolation, lack of consistent case definitions and reliance on case reporting $[25,26]$. The aim of this study was to describe the clinical spectrum of F. nucleatum isolates in an urban county hospital setting. 


\section{Patients and Methods}

Clinical and microbiological data were obtained from both Ben Taub Hospital and Lyndon B. Johnson Hospital. Ben Taub General is located in Houston, Texas within the Texas Medical Center. It is operated by the Harris Health System and is staffed by faculty, residents and students from Baylor College of Medicine. It is one of three level one trauma centers in southeast Texas. It has 586 licensed beds and averages 24,000 patient admissions in a year.

Lyndon B. Johnson Hospital is located in Houston on the northeast side. It is also operated by Harris Health System and is staffed by faculty, residents and students from University of Texas Health Science Center at Houston. It is a level three trauma center. It has 328 licensed beds and averages 16,000 medicine admissions in a year.

Consecutive isolates of $F$. nucleatum from any site were identified from the hospital laboratory database from 1 January, 2002 to 31 December, 2011. Hospital and laboratory policy throughout the period of the study was to recommend aerobic and anaerobic cultures for adult patients. Clinical specimens were submitted for aerobic and anaerobic cultures after transfer in sterile containers to the hospital laboratories which are on-site. Identification of F. nucleatum was based on typical colony morphologies without other biochemical testing. Antibiotic susceptibility testing was not carried out and so beta-lactamase production in clinical isolates could not be assessed.

A single reviewer classified primary site and localized/invasive infection in each case. The following clinical and epidemiological data were collected from the electronic medical record: age, gender, duration of hospitalization, clinical presentation on admission, infection site, treatment, final outcome and risk factors (diabetes mellitus type I/II, liver and heart disease, malignancy, immune deficiency, injuries/lesions or wounds). In addition, the following microbiological data were also collected: clinical specimen type and other species isolated from the same specimen. Survival to 30 days from the date of the first positive microbiological specimen was determined from the clinical record. Infections were classified by primary site into six groups. These were: 1) brain; 2) head and neck; 3) abdomino-pelvic; 4) skin and soft tissue; 5) pleuro-pulmonary; and 6) blood. Data were entered into a Microsoft Excel spreadsheet. Descriptive analysis of epidemiological characteristics of all patients was performed (Figure 1). The protocol was approved by the Baylor College of Medicine Institutional Review Board and Harris Health System Office of Research. All data were collected without any identifiable information.

\section{Location of Fusibacterium nucleatum Infections}

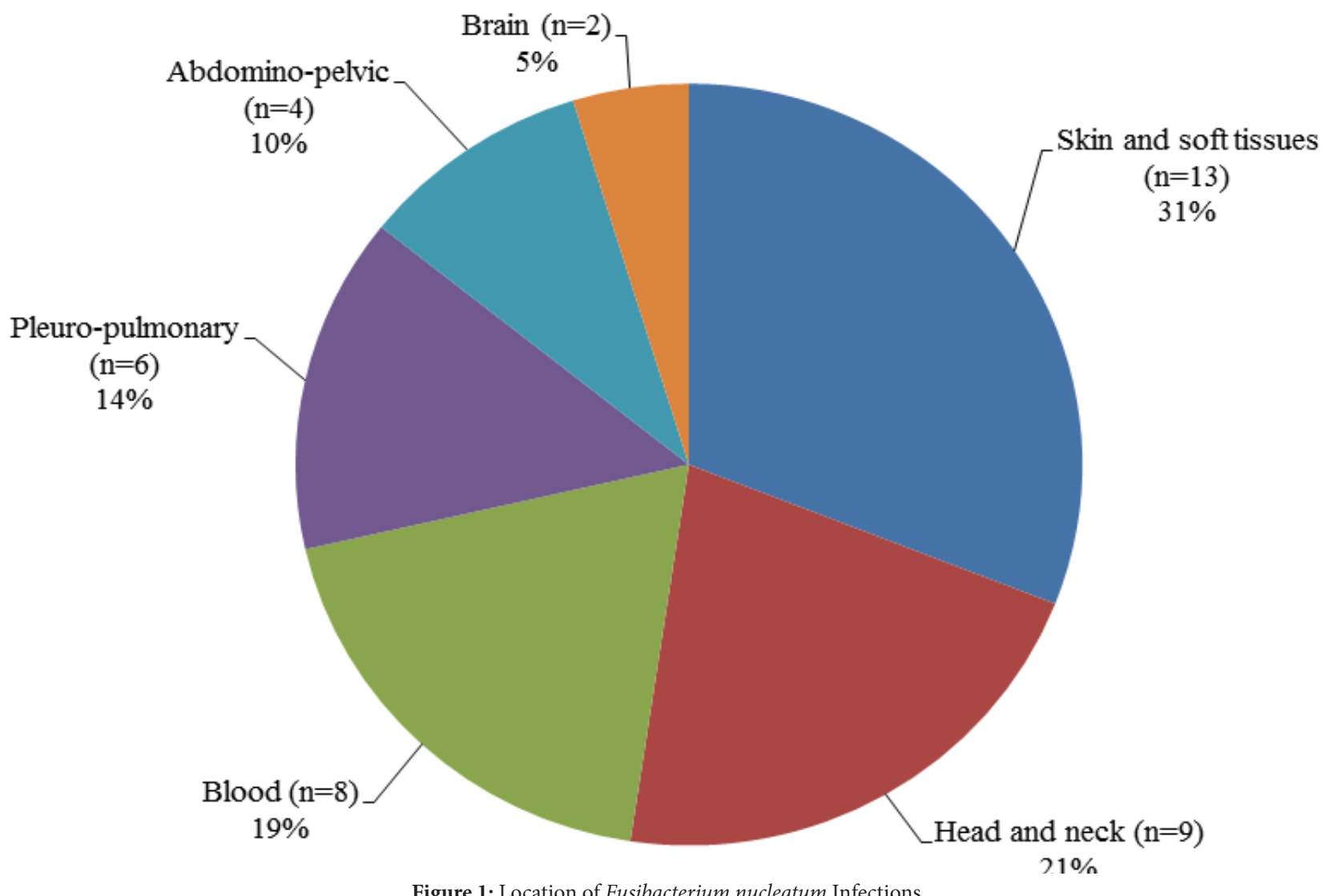

Figure 1: Location of Fusibacterium nucleatum Infections 


\section{Results}

\section{Epidemiology of F.nucleatum isolation}

The overall incidence of patients with culture isolation of F. nucleatum during the study period was $0.0113 \%$ (45 patients from 400,000 patient admissions). The mean age was 45.5 (SD 14.5) and median age was 45 years (IQR 33.5-53.8). 6.3\% were identified as immunocompromised. A predilection for males was also noted in our study, with males representing $70 \%$ of the study subjects. It was also noted $20 \%$ of the identified subjects used illicit drugs and 35\% used alcohol. Of the 40 patients identified, 38 (95\%) survived at least 30 days after discharge from the hospital. The one identified death was related to a brain abscess (Table 1).

\begin{tabular}{|l|l|}
\hline \multicolumn{2}{|c|}{$\begin{array}{c}\text { Demographics of Patients with Fusobacterium } \\
\text { nucleatum Infections }\end{array}$} \\
\hline Gender & $70 \%(28)$ \\
\hline Male & $30 \%(12)$ \\
\hline Female & \\
\hline Age & $70 \%(28)$ \\
\hline$<=50$ & $30 \%(12)$ \\
\hline$>\mathbf{5 0}$ & $45.5(\mathrm{SD} 14.5)$ \\
\hline Mean Age & $45(\mathrm{IQR} 33.5-53.8)$ \\
\hline Median Age & \\
\hline Past Medical History & $57.5 \%(23)$ \\
\hline History of smoking & $35 \%(14)$ \\
\hline History of alcohol use & $20 \%(8)$ \\
\hline History of illicit drug use & $35 \%(14)$ \\
\hline Hypertension & $17.5 \%(7)$ \\
\hline Diabetes Mellitus & $7.5 \%(3)$ \\
\hline HIV & \\
\hline
\end{tabular}

Table 1: Demographics of patients found to have F. nucleatum infections

\section{Clinical presentation of F. nucleatum isolation}

There were 48 cultures identified in 45 patients with the remaining 3 cultures being from placental specimens. Clinical data for 5 patients was unavailable and were not included in this analysis. There were 42 cultures of F. nucleatum isolated from the remaining 40 subjects. Infections in this group were categorized as follows: skin and soft tissues - 13 isolates (31.0\%), head and neck - 9 isolates (21.4\%), blood -8 isolates (19.0\%), pleuro-pulmonary - 6 isolates (14.3\%), abdomino-pelvic -4 isolates (9.5\%), and brain -2 isolates $(4.8 \%)$.

Head and neck isolates included submandibular and dental abscesses, otitis media with periauricular cellulitis, wound infection following trauma and head and neck surgery; one isolate was recovered from a tonsillar biopsy performed for diagnosis of a malignancy. All except 2 patients were treated with clindamycin or metronidazole, either singly or in combination with amoxicillin or a cephalosporin. The doses of clindamycin were most often $600 \mathrm{mg}$ intravenous every eight hours and then $600 \mathrm{mg}$ orally three times daily, there were ocassional variations to include $900 \mathrm{mg}$ intravenous every eight hours and $300-450 \mathrm{mg}$ orally three times daily. The doses of metronidazole used were 500mg intravenous every eight hours and then 500mg orally three times daily. The patient with periauricular cellulitis was treated with vancomycin along with a mastoidectomy. One of the patients with a submandibular abscess was treated with amoxicillin/clavulanate after incision and drainage and identification of the organism.

Blood cultures were positive for F. nucleatum in eight patients. One was in a patient with an infected permacath and also grew methicillin-resistant Staphylococcus aureus. One isolate was obtained from the blood of a psychiatric patient seen only in the emergency department for asthma exacerbation; no follow-up information was available. Two of the isolates were obtained from the blood of patients admitted for syncope and seizure. Neither patient remained as an inpatient long enough for the culture data to become available and received no specific therapy. One patient had HIV infection; the other had only sickle cell disease and also grew Gemella morbillorum.

Four pleuro-pulmonary isolates were cultured from empyemas. Among these patients with empyema, 2 were incarcerated and 2 had concomitant tuberculosis infection. There was no identified simultaneous oral infection or dysphagia in these patients, but it often was not specifically addressed. The 2 remaining pleuro-pulmonary infections were complicated pneumonias. Of these, 1 patient refused treatment and the other was a 59-year-old woman with a history of hypertension, hepatitis $\mathrm{C}$ and chronic obstructive pulmonary disease who presented in hypoxic respiratory failure. This patient was found to have pneumonia based on bilateral patchy opacities on chest radiograph. Her blood cultures obtained on admission grew F. nucleatum. She received multiple broad spectrum antibiotics, including anaerobic coverage; however, she continued to deteriorate and died 18 days after admission. 
Abdominal isolates were recovered from perirectal and liver abscesses, as well as a stomach aspirate. The patient with a liver abscess had comorbid alcoholic cirrhosis, acute on chronic pancreatitis with pseudocyst and uncontrolled diabetes mellitus with an $\mathrm{HgA} 1 \mathrm{C}$ of $13.9 \%$. The stomach aspirate was obtained during a diagnostic procedure for gastric adenocarcinoma. The patient underwent a total gastrectomy and treatment with ceftriaxone and metronidazole. The liver abscess and one perirectal abscess were treated with a flouroquinolone in combination with metronidazole. The remaining perirectal abscess was treated with surgical intervention alone. Skin and soft tissue isolates came from 4 abdominal incision site infections, 4 extremity infections, 2 of whom abused parenteral drugs, a decubitus ulcer, and a thoracoabdominal wall abscess of unknown etiology in a severely psychotic patient; three were breast abscesses in women not reporting to be breastfeeding. Most cases were treated with anaerobic antibiotics, but all were treated surgically. There were no identified relapses.

One brain isolate was identified in a patient with comorbid laryngeal cancer. This patient also had heart disease, hepatitis $\mathrm{C}$ and end-stage renal disease and succumbed to death. The other brain isolate was in a 50-year-old male with no comorbid conditions that had undergone unknown dental surgery in Mexico several weeks prior to his presentation. He recovered fully.

\section{Discussion}

We conducted a 10 year retrospective population based study at two acute care urban hospitals. Previous reports have mainly focused on invasive Fusobacterium infections in tertiary-care centers, whereas we attempted to describe the epidemiology of primary site isolation in two county hospitals. Forty patients with culture documented F. nucleatum associated infections from 2002 to 2011 were included in this study. In previous reports, F. nucleatum was found primarily in older patients with chronic medical conditions and/or immunocompromised state and was associated with a mortality of approximately $10 \%[24,26,27]$. In contrast, our study identified the mean age at 45.5 years with minimal to no concomitant chronic conditions and only two deaths were documented. Most US case series focus on fusobacterium bacteremia, whereas we attempted to describe all infections associated with culture positive fusobacterium [3-5,8,9,11]. In the limited reports that have described non-bacteremic infections, either pleuro-pulmonary or intra-abdominal sites have been identified as the most common source $[24,26,27]$. However, in our identified patient population, F. nucleatum was isolated from a wound or abscess in $77.5 \%$, only one of which had associated bacteremia. $100 \%$ of the patients with skin or soft tissue infection underwent surgical intervention. Our high rate of skin and soft tissue infections associated with early surgical intervention may account for our low rate of bacteremia. The prevalent risk factors identified in skin and soft tissue infections were surgical site wound and drug or alcohol use. There were no reports of human or animal inoculation of the infection site, although it was suspected in the patient with a thoraco-abdominal wall abscess of unknown origin.

It was also noted that $84.6 \%$ of the skin and soft tissue infections also received anaerobic antibiotic coverage. Adequate details of antimicrobial therapy provided were identified in all but 2 of the study population. Initial antibiotic therapy consisted of anaerobic coverage in $42.5 \%$ of cases and increased to $75 \%$ after F. nucleatum was isolated. Average length of hospital stay was 7.2 days after extracting 2 patients that stayed $>30$ days. The average length of time prior to F. nucleatum culture positivity was 8.9 days, with 3 reported after 20 days. Thus, the majority of antibiotic decisions were made prior to identification. On discharge $92 \%$ of patients received antimicrobial therapy. Clindamycin or metronidazole comprised the majority of the antibiotics prescribed on discharge; 2 patients received amoxicillin/clavulanate, 2 received TMP-SMX and one patient received gatifloxacin. All but two patients recovered fully. Recent studies have described developing antibiotic resistance in Fusobacterium species [28-31]. One study reported variable in vitro response of F. nucleatum to TMP-SMX [32]. Multicenter studies have also reported resistance to clindamycin in Fusobacterium species varying between 4 and 33\% [28-31,33,34]. However, despite the increasing evidence suggesting reduced susceptibility or resistance to the commonly used empiric antibiotics, none of our identified patients with skin or soft tissue infection had a relapse of infectious signs or symptoms within 30 days of discharge. Again, this may also be in part due to early surgical intervention (Table 2).

\begin{tabular}{|c|c|c|c|c|c|c|}
\hline \multicolumn{7}{|c|}{ Clinical Manifestations of Fusobacterium nucleatum Infections and Treatment } \\
\hline Location of infection & Type of infection & $\begin{array}{l}\text { No. of } \\
\text { cases }\end{array}$ & $\begin{array}{l}\text { Additional microbes } \\
\text { isolated other than } \\
\text { Fusobacterium } \\
\text { nucleatum }\end{array}$ & $\begin{array}{c}\text { Treatment } \\
\text { administered } \\
\text { prior to microbe } \\
\text { identification }\end{array}$ & $\begin{array}{c}\text { Treatment } \\
\text { administered } \\
\text { after microbe } \\
\text { identification }\end{array}$ & $\begin{array}{l}\text { Treatment other } \\
\text { than antibiotics }\end{array}$ \\
\hline \multirow{2}{*}{ Brain } & \multirow[t]{2}{*}{ Brain abscesses } & \multirow[t]{2}{*}{2} & (a)- & $\begin{array}{l}\text { Vancomycin, } \\
\text { piperacillin/ } \\
\text { tazobactam }\end{array}$ & $\begin{array}{l}\text { Cefepime, } \\
\text { metronidazole }\end{array}$ & Burr Hole \\
\hline & & & (b)- & $\begin{array}{l}\text { Penicillin G, } \\
\text { metronidazole }\end{array}$ & $\begin{array}{l}\text { Penicillin G, } \\
\text { metronidazole }\end{array}$ & $\mathrm{I} \& \mathrm{D}$ \\
\hline \multirow[b]{2}{*}{ Head and Neck } & \multirow{2}{*}{$\begin{array}{l}\text { Submandibular } \\
\text { abscesses }\end{array}$} & \multirow[b]{2}{*}{3} & $\begin{array}{l}\text { (a) a-hemolytic } \\
\text { Streptococcus }\end{array}$ & - & $\begin{array}{l}\text { Amoxicillin/ } \\
\text { Clavulanate }\end{array}$ & $\mathrm{I} \& \mathrm{D}$ \\
\hline & & & $\begin{array}{l}\text { (b) Prevotella } \\
\text { melaninogenica and } \\
\text { Prevotella buccae }\end{array}$ & Clindamycin & Clindamycin & $\mathrm{I} \& \mathrm{D}$ \\
\hline
\end{tabular}




\begin{tabular}{|c|c|c|c|c|c|c|}
\hline \multicolumn{7}{|c|}{ Clinical Manifestations of Fusobacterium nucleatum Infections and Treatment } \\
\hline Location of infection & Type of infection & $\begin{array}{l}\text { No. of } \\
\text { cases }\end{array}$ & $\begin{array}{c}\text { Additional microbes } \\
\text { isolated other than } \\
\text { Fusobacterium } \\
\text { nucleatum }\end{array}$ & $\begin{array}{c}\text { Treatment } \\
\text { administered } \\
\text { prior to microbe } \\
\text { identification }\end{array}$ & $\begin{array}{c}\text { Treatment } \\
\text { administered } \\
\text { after microbe } \\
\text { identification }\end{array}$ & $\begin{array}{l}\text { Treatment other } \\
\text { than antibiotics }\end{array}$ \\
\hline & & & $\begin{array}{l}\text { (c) Prevotella } \\
\text { melaninogenica and } \\
\text { Prevotella intermedia }\end{array}$ & $\begin{array}{l}\text { Cefazolin, } \\
\text { Clindamycin }\end{array}$ & $\begin{array}{l}\text { Cefazolin, } \\
\text { Metronidazole }\end{array}$ & $\mathrm{I} \& \mathrm{D}$ \\
\hline & Dental abscesses & 2 & (a) - & Clindamycin & Clindamycin & $\mathrm{I} \& \mathrm{D}$ \\
\hline & & & $\begin{array}{l}\text { (b) Prevotella } \\
\text { intermedia, Prevotella } \\
\text { buccae, a-hemolytic } \\
\text { Streptococcus, CONS }\end{array}$ & Ceftriaxone & $\begin{array}{l}\text { Clindamycin, } \\
\text { Ampicillin/Sulbactam }\end{array}$ & Wash out \\
\hline & $\begin{array}{l}\text { Otitis media with per- } \\
\text { iauricular cellulitis }\end{array}$ & 1 & (a) - & Vancomycin & Vancomycin & Mastoidectomy \\
\hline & $\begin{array}{l}\text { Left mandibular frac- } \\
\text { ture with cellulitis }\end{array}$ & 1 & (a) - & - & Clindamycin & Buccal exploration \\
\hline & $\begin{array}{l}\text { Tonsillar biopsy for } \\
\text { malignancy }\end{array}$ & 1 & (a) - & Clindamycin & Clindamycin & Biopsy \\
\hline & Buccal infection & 1 & (a) - & $\begin{array}{l}\text { Vancomycin, } \\
\text { cefepime, acyclovir }\end{array}$ & $\begin{array}{l}\text { Metronidazole, acy- } \\
\text { clovir, ciprofloxacin, } \\
\text { fluconazole }\end{array}$ & Excision \\
\hline \multirow{4}{*}{ Abdomino-pelvic } & \multirow[b]{2}{*}{ Perirectal abscesses } & \multirow[b]{2}{*}{2} & $\begin{array}{l}\text { (a) Bacteroides } \\
\text { thetaiotaomicron, } \\
\text { E.coli, GDE }\end{array}$ & - & $\begin{array}{l}\text { Metronidazole, fluo- } \\
\text { roquinolone }\end{array}$ & $\mathrm{I} \& \mathrm{D}$ \\
\hline & & & $\begin{array}{l}\text { (b) Peptostrepto } \\
\text { coccus anaerobius, } \\
\gamma \text {-hemolytic } \\
\text { Streptococcus. }\end{array}$ & - & - & $I \& D$ \\
\hline & Liver abscesses & 1 & $\begin{array}{l}\text { (a) MSSA, CONS, } \\
\text { Actinomyces spp., } \\
\text { GDE, Prevotella oris } \\
\text { and Prevotella oralis }\end{array}$ & Ampicillin/Sulbactam & $\begin{array}{l}\text { Ciprofloxacin, metro- } \\
\text { nidazole }\end{array}$ & $\begin{array}{l}\text { I\&D (Interventional } \\
\text { radiology guided) }\end{array}$ \\
\hline & Stomach aspirate & 1 & $\begin{array}{l}\text { (a) Klebsiella pneu- } \\
\text { moniae }\end{array}$ & $\begin{array}{l}\text { Ceftriaxone, } \\
\text { metronidazole }\end{array}$ & $\begin{array}{l}\text { Ceftriaxone and } \\
\text { metronidazole }\end{array}$ & Cancer resection \\
\hline \multirow[t]{6}{*}{ Skin and Soft tissue } & $\begin{array}{l}\text { Abdominal incision } \\
\text { site infection }\end{array}$ & 4 & $\begin{array}{l}\text { (a) Bacteroides fragilis, } \\
\text { Enterobacer aerogenes, } \\
\text { Morganella morganii, } \\
\text { Citrobacter freundii, } \\
\text { GDE }\end{array}$ & $\begin{array}{l}\text { Ciprofloxacin, } \\
\text { metronidazole }\end{array}$ & $\begin{array}{l}\text { Cefepime, imipenem, } \\
\text { rifampin, vancomycin }\end{array}$ & $\begin{array}{l}\text { Emergency } \\
\text { exploratory } \\
\text { laparotomy }\end{array}$ \\
\hline & & & $\begin{array}{l}\text { (b) Bacteroides } \\
\text { fragilis, E.coli, } \\
\gamma \text {-hemolytic } \\
\text { Streptococcus, } \\
\text { Corynebacterium spp., } \\
\text { Candida albicans }\end{array}$ & $\begin{array}{l}\text { Ceftriaxone, } \\
\text { ciprofloxacin, } \\
\text { metronidazole, } \\
\text { vancomycin }\end{array}$ & $\begin{array}{l}\text { Ceftriaxone, } \\
\text { ciprofloxacin, } \\
\text { metronidazole, } \\
\text { vancomycin }\end{array}$ & $\begin{array}{l}\text { Resection of } \\
\text { abdominal tissue, } \\
\text { multiple washouts } \\
\text { and wound vac } \\
\text { exchange. }\end{array}$ \\
\hline & & & $\begin{array}{l}\text { (c) Bacteroides } \\
\text { uniformis, Prevotella } \\
\text { loescheil, E.coli, } \\
\gamma \text {-hemolytic } \\
\text { Streptococcus, GDE, } \\
\beta \text {-Streptococcus } \\
\end{array}$ & $\begin{array}{l}\text { Ciprofloxacin, } \\
\text { metronidazole }\end{array}$ & $\begin{array}{l}\text { Ciprofloxacin, } \\
\text { metronidazole }\end{array}$ & Debridement \\
\hline & & & (d) unknown & $\begin{array}{l}\text { "antibiotics" without } \\
\text { further specification }\end{array}$ & $\begin{array}{l}\text { "antibiotics" without } \\
\text { further specification }\end{array}$ & $I \& D$ \\
\hline & Extremity infection & 4 & $\begin{array}{l}\text { (a) Prevotella } \\
\text { melaninogenica, } \\
\text { a-hemolytic } \\
\text { Streptococcus, } \\
\text { Corynebacterium spp. }\end{array}$ & $\begin{array}{l}\text { Vancomycin, } \\
\text { clindamycin }\end{array}$ & $\begin{array}{l}\text { TMP-SMX, } \\
\text { amoxicillin }\end{array}$ & $\mathrm{I} \& \mathrm{D}$ \\
\hline & & & $\begin{array}{l}\text { (b) } \\
\text { Corynebacterium } \\
\text { spp., GBS, } \\
\text { Peptostreptococcus } \\
\text { anaerobius }\end{array}$ & Cefazolin & TMP-SMX & Amputation \\
\hline
\end{tabular}




\begin{tabular}{|c|c|c|c|c|c|c|}
\hline \multicolumn{7}{|c|}{ Clinical Manifestations of Fusobacterium nucleatum Infections and Treatment } \\
\hline \multirow[t]{8}{*}{ Location of infection } & Type of infection & $\begin{array}{l}\text { No. of } \\
\text { cases }\end{array}$ & $\begin{array}{l}\text { Additional microbes } \\
\text { isolated other than } \\
\text { Fusobacterium } \\
\text { nucleatum }\end{array}$ & $\begin{array}{c}\text { Treatment } \\
\text { administered } \\
\text { prior to microbe } \\
\text { identification }\end{array}$ & $\begin{array}{c}\text { Treatment } \\
\text { administered } \\
\text { after microbe } \\
\text { identification }\end{array}$ & $\begin{array}{l}\text { Treatment other } \\
\text { than antibiotics }\end{array}$ \\
\hline & & & $\begin{array}{l}\text { (c)GBS, orynebacte- } \\
\text { rium spp., Peptostrep- } \\
\text { tococcus micros }\end{array}$ & $\begin{array}{l}\text { Cefazolin, } \\
\text { gatifloxacin }\end{array}$ & Cefalexin & $I \& D$ \\
\hline & & & $\begin{array}{l}\text { (d) No information } \\
\text { identified }\end{array}$ & $\begin{array}{l}\text { Cefepime, } \\
\text { vancomycin }\end{array}$ & $\begin{array}{l}\text { Vancomycin, } \\
\text { metronidazole }\end{array}$ & Debridement \\
\hline & Decubitus ulcer & 1 & $\begin{array}{l}\text { (a) Bacteroides } \\
\text { distasonis, Bacteroides } \\
\text { thetaiotaomicron }\end{array}$ & $\begin{array}{l}\text { Vancomycin, } \\
\text { metronidazole, } \\
\text { ceftriaxone }\end{array}$ & $\begin{array}{l}\text { Vancomycin, } \\
\text { metronidazole, } \\
\text { ceftriaxone }\end{array}$ & I\&D \\
\hline & $\begin{array}{l}\text { Thoracoabdominal } \\
\text { wall abscess of } \\
\text { unknown etiology }\end{array}$ & 1 & $\begin{array}{l}\text { (a) No information } \\
\text { available }\end{array}$ & $\begin{array}{l}\text { No information } \\
\text { available }\end{array}$ & $\begin{array}{l}\text { No information } \\
\text { available }\end{array}$ & $\begin{array}{l}\text { Splenectomy, thora- } \\
\text { cotomy }\end{array}$ \\
\hline & Breast abscesses & 3 & (a) - & - & - & $\mathrm{I} \& \mathrm{D}$ \\
\hline & & & (b) - & Vancomycin, efepime & TMP-SMX & $\mathrm{I} \& \mathrm{D}$ \\
\hline & & & $\begin{array}{l}\text { (c) } \beta \text {-hemolytic } \\
\text { Streptococcus spp. }\end{array}$ & - & $\begin{array}{l}\text { Gatifloxacin, } \\
\text { metronidazole }\end{array}$ & $\mathrm{I} \& \mathrm{D}$ \\
\hline \multirow[t]{6}{*}{$\begin{array}{l}\text { Pleuro-pulmonary } \\
\text { infections }\end{array}$} & Empyema & 4 & $\begin{array}{l}\text { (a) No information } \\
\text { available }\end{array}$ & - & - & Thoracotomy \\
\hline & & & $\begin{array}{l}\text { (b) No information } \\
\text { available }\end{array}$ & Cefdinir & $\begin{array}{l}\text { Amoxicillin/ } \\
\text { Clavulanate }\end{array}$ & Thoracotomy \\
\hline & & & (c) - & $\begin{array}{l}\text { Ceftriaxone, } \\
\text { azithromycin }\end{array}$ & $\begin{array}{l}\text { Ceftriaxone, } \\
\text { clindamycin }\end{array}$ & Thoracotomy \\
\hline & & & (d)CONS & $\begin{array}{l}\text { Vancomycin, } \\
\text { cefepime, clindaycin }\end{array}$ & $\begin{array}{l}\text { TMP-SMX, } \\
\text { clindamycin, rifampin }\end{array}$ & Chest tube drainage \\
\hline & Pneumonia & 2 & $\begin{array}{l}\text { (a) Peptostreptococcus } \\
\text { sacchrolyticus }\end{array}$ & - & - & Refused treatment \\
\hline & & & (b) - & $\begin{array}{l}\text { Vancomycin, } \\
\text { Cefepime, } \\
\text { Ceftriaxone, } \\
\text { clindamycin, TMP- } \\
\text { SMX, Azithromycin, }\end{array}$ & $\begin{array}{l}\text { Vancomycin, } \\
\text { Fluconazole, } \\
\text { Cefepime, imipenem/ } \\
\text { cilastin, moxifloxacin, } \\
\text { amphotericin B }\end{array}$ & - \\
\hline \multirow[t]{8}{*}{ Blood } & Blood-borne & 8 & (a) MRSA & Vancomycin & - & Permacath removal \\
\hline & & & (b) - & $\begin{array}{l}\text { Vancomycin, } \\
\text { Cefepime, } \\
\text { Ceftriaxone, } \\
\text { clindamycin, TMP- } \\
\text { SMX, Azithromycin, }\end{array}$ & $\begin{array}{l}\text { Vancomycin, } \\
\text { Fluconazole, } \\
\text { Cefepime, imipenem/ } \\
\text { cilastin, moxifloxacin, } \\
\text { amphotericin B }\end{array}$ & - \\
\hline & & & (c) - & $\begin{array}{l}\text { Vancomycin, } \\
\text { ceftriaxone }\end{array}$ & Metronidazole & - \\
\hline & & & (d) - & Gatifloxacin & Gatifloxacin & - \\
\hline & & & (e) - & $\begin{array}{l}\text { Vancomycin, } \\
\text { cefepime, acyclovir }\end{array}$ & $\begin{array}{l}\text { Metronidazole, acy- } \\
\text { clovir, ciprofloxacin, } \\
\text { fluconazole }\end{array}$ & Excision \\
\hline & & & $\begin{array}{l}\text { (f) Gemella morbil- } \\
\text { brun, Peptostreptococ- } \\
\text { cus spp. }\end{array}$ & - & Amoxicillin/clavuaate & \\
\hline & & & (g) M. tuberculae & - & $\begin{array}{l}\text { Rifampin, } \\
\text { ethambutol, isoniazid, } \\
\text { pyrazinamide, } \\
\text { metronidazole }\end{array}$ & - \\
\hline & & & (h) - & $\begin{array}{l}\text { Azithromycin, ceftri- } \\
\text { axone }\end{array}$ & - & - \\
\hline
\end{tabular}

Table 2: Clinical Manifestations of Fusibacterium nucleatum Infections and Treatment

Acronyms used: (CONS) Coagulase-negative staphylococcus aureus; (GBS) Group B Streptococcus; (GDS) Group D Streptococcus; (GDE) Group D Enterococcus; (MSSA) I\&D Incision and drainage; Methicillin-sensitive Staphylococcus aureus; (MRSA) Methicillin-resistant Staphylococcus aureus; (spp) species; (TMP-SMX) - Trimethoprim-sulfamethoxazole. 


\section{Conclusion}

We have reported a series of 40 cases of Fusobacterium nucleatum infections from a general county hospital population in an urban setting. In our study, skin and soft tissue infections were the most frequent sites of F. nucleatum isolation. This result is different from previous reports demonstrating respiratory tract infections, especially pneumonia, as the predominant identified source, followed by intra-abdominal sites $[5,8,9,24,26]$. There was no association noted with an immunocompromised state, which is also in contrast to previous reports, citing malignancy as the most common associated immunocompromised state [5,8,9]. Clindamycin and metronidazole were the two most frequently chosen drugs for antimicrobial therapy. It is also important to note, almost all cases involved some form of surgical treatment aside from antibiotic therapy.

\section{Acknowledgement}

We would like to acknowledge Dr. Richard Hamill.

\section{References}

1. Bolstad AI, Jensen HB, Bakken V (1996) Taxonomy, biology and periodontal aspects of Fusobacterium nucleatum. Clinical Microbiol Rev 9: 55-71.

2. Batty A, Wren MW (2005) Prevalence of Fusobacterium necrophorum and other upper respiratory tract pathogens isolated from throat swabs. Br J Biomed Sci 62: 66-70.

3. Bourgault AM, Lamothe F, Dolcé P, Saint-Jean L, Saint-Antoine P (1997) Fusobacterium bacteremia: clinical experience with 40 cases. Clin Infect Dis 25: S181-3.

4. Brook I (2010) The role of anaerobic bacteria in bacteremia. Anaerobe 16: 183-9.

5. Yang CC, Ye JJ, Hsu PC, Chang HJ, Cheng CW, et al. (2011) Characteristics and outcomes of Fusobacterium nucleatum bacteremia--a 6-year experience at a tertiary care hospital in northern Taiwan. Diagn Microbiol Infect Dis 70: 167-74.

6. Jousimies-Somer H, Savolainen S, Mäkitie A, Ylikoski J (1993) Bacteriologic findings in peritonsillar abscesses in young adults. Clin Infect Dis 16: S292-8.

7. Nadkarni MD, Verchick J, O’Neill JC (2005) Lemierre syndrome. J Emerg Med 28: 297-99.

8. Afra K, Laupland K, Leal J, Lloyd T, Gregson D (2013) Incidence, risk factors, and outcomes of Fusobacterium species bacteremia. BMC Infect Dis 13: 264.

9. Blairon L, De Gheldre Y, Delaere B, Sonet A, Bosly A, et al. (2006) A 62-month retrospective epidemiological survey of anaerobic bacteraemia in a university hospital. Clin Microbiol Infect 12: 527-32.

10. Smith GR, Thornton EA (1993) Pathogenicity of Fusobacterium necrophorum strains from man and animals. Epidemiol Infect 110: 499-506.

11. Su CP, Huang PY, Yang CC, Lee MH (2009) Fusobacterium bacteremia: clinical significance and outcomes. J Microbiol Immunol Infect 42: 336-42.

12. Bennett KW, Eley A (1993) Fusobacteria: new taxonomy and related diseases. J Med Microbiol 39: 246-54.

13. Huggan PJ, Murdoch DR (2008) Fusobacterial infections: Clinical spectrum and incidence of invasive disease. J Infect 57: 283-9.

14. Kornman KS, Page RC, Tonetti MS (1997) The host response to the microbial challenge in periodontitis: assembling the players. Periodontol 14: $33-53$.

15. Loos BG, Craandijk J, Hoek FJ, Wertheim-van Dillen PM, van der Velden U (2000) Elevation of systemic markers related to cardiovascular diseases in the peripheral blood of periodontitis patients. J Periodontol 71: 1528-34.

16. Scannapieco FA. Periodontal inflammation: from gingivitis to systemic disease? Compend Cont Educ Dent 25: 16-25.

17. Amano A (2009) Bacterial adhesins to host components in periodontitis. Periodontology 52: 12-37.

18. Baron EJ, Citron DM (1997) Anaerobic identification flowchart using minimal laboratory resources. Clin Infect Dis 25 : S143-6.

19. Tanner AC, Strzempko MN, Belsky CA, McKinley GA (1985) API ZYM and API An-Ident reactions of fastidious oral gram-negative species. J Clin Microbiol 22: $333-5$.

20. Sigge A, Essig A, Wirths B, Fickweiler K, Kaestner N, et al. (2007) Rapid identification of Fusobacterium nucleatum and Fusobacterium necrophorum by fluorescence in situ hybridization. Diagn Microbiol Infect Dis 58: 255-9.

21. Al-Haroni M, Skaug N, Bakken V, Cash P (2008) Proteonomic analysis of ampicillin-resistant oral Fusobacterium nucleatum. Oral Microbiol Immunol 23: $36-42$.

22. Shilnikova II, Dmitrieva NV (2014) Evaluation of antibiotic susceptibility of Bacteroides, Prevotella and Fusobacterium species isolated from patients of the N. N. Blokhin Cancer Research Center, Moscow, Russia. Anaerobe doi: 10.1016/j.anaerobe.2014.08.003.

23. Steeves CH, Potrykus J, Barnett DA, Bearne SL (2011) Oxidative stress response in the opportunistic oral pathogen Fusobacterium nucleatum. Proteomics 11: 2027-37.

24. Ngo JT, Parkins MD, Gregson DB, Pitout JD, Ross T, et al. (2013) Population-based assessment of the incidence, risk factors, and outcomes of anaerobic bloodstream infections. Infection 41: 41-8.

25. Ewald C, Kuhn S, Kalff R (2006) Pyogenic infections of the central nervous system secondary to dental affections--a report of six cases. Neurosurg Rev 29: 163-6.

26. Goldberg EA, Venkat-Ramani T, Hewit M, Bonilla HF (2013) Epidemiology and clinical outcomes of patients with Fusobacterium bacteraemia. Epidemiol Infect 141: 325-9.

27. Murray PR, Baron EJ, Jorgensen JH, Landry ML (2007) Bacteroides, porphyromonas, prevotella, fusobacterium, and other anaerobic gram-negative rods. Manual of Clinical Microbiology ( $9^{\text {th }}$ edn) ASM Press, Washington DC.

28. Aldridge KE, Ashcraft D, Cambre K, Pierson CL, Jenkins SG, et al. (2001) Multicenter survey of the changing in vitro antimicrobial susceptibilities of clinical isolates of Bacteroides fragilis group, Prevotella, Fusobacterium, Porphyromonas, and Peptostreptococcus species. Antimicrob Agents Chemother 45: 1238-43.

29. Brazier JS, Hall V, Morris TE, Gal M, Duerden BI (2003) Antibiotic susceptibilities of Gram-positive anaerobic cocci: results of a sentinel study in England and Wales. J Antimicrob Chemother 52: 224-8.

30. Brazier J, Chmelar D, Dubreuil L, Feierl G, Hedberg M, et al. (2008) European surveillance study on antimicrobial susceptibility of Gram-positive anaerobic cocci. Int J Antimicrob Agents 31: 316-20.

31. Citron DM, Goldstein EJC, Vreni Merriam C, Lipsky BA, Abramson MA (2007) Bacteriology of moderate-to-severe diabetic foot infections and in vitro activity of antimicrobial agents. J Clin Microbiol 45: 2819-28.

32. Wust J, Wilkins TD (1978) Susceptibility of anaerobic bacteria to sulfamethoxazole/trimethoprim and routine susceptibility testing. Antimicrob Agents Chemother 14: 384-90.

33. García-Rodríguez JA, García-Sánchez JE, Muñoz-Bellido JL (1995) Antimicrobial resistance in anaerobic bacteria: current situation. Anaerobe 1: 69-80.

34. Papaparaskevas J, Pantazatou A, Katsandri A, Legakis NJ, Avlamis A, et al. (2005) Multicentre survey of the in-vitro activity of seven antimicrobial agents, including ertapenem, against recently isolated Gram-negative anaerobic bacteria in Greece. Clin Microbiol Infect 11: 820-4. 


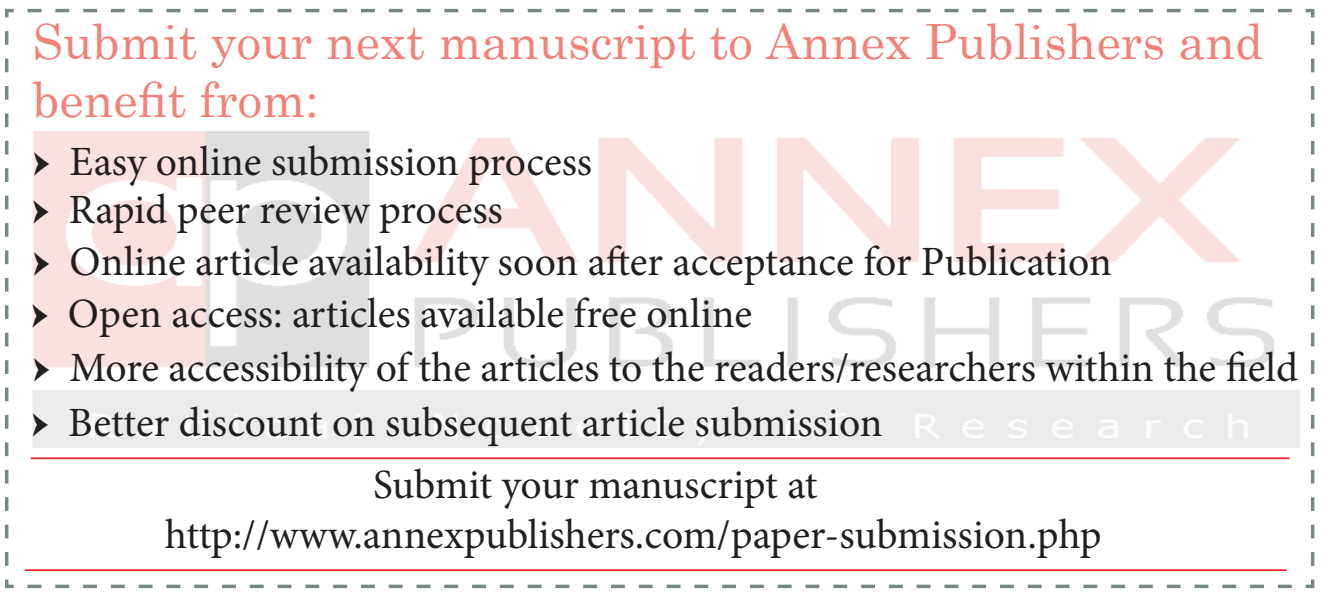

